\title{
An Efficient Destination Sequenced Distance Vector for Mobility based Asymmetric Networks
}

\author{
Palak Girdhar \\ Assistant Professor \\ CSE Departent \\ BPIT, Delhi, India
}

\author{
Anshu Khatri \\ Student, CSE Department \\ Amrita School of Engineering \\ Coimbatore
}

\author{
Tanu Sharma \\ Assistant Professor \\ CSE Department \\ BPIT, Delhi, India
}

\begin{abstract}
The mobile ad hoc network technology is a wireless interconnection of mobile devices and has dynamic topology. It does not have any fixed network infrastructure and the disparity in the transmission range of different nodes can result into the unidirectional asymmetric links in the network. It has adverse impacts on the overall performance of most of the routing protocols which consider all the wireless links in the network as bidirectional. Hence, a new model is proposed in this paper. It uses the routing table to store the information about the outneighbours and in-neighbours for a node which helps to determine the shortest path to the destination node as well as the reverse path from the unidirectional out-neighbour to the node itself. The paper investigates the existing methods and concludes that the new U-DSDV works efficiently in unidirectional links.
\end{abstract}

\section{Keywords}

DSDV, MANET, unidirectional links, asymmetric network

\section{INTRODUCTION}

Mobile ad hoc network (MANET) is a collection of mobile nodes (MN) which do not require any kind of infrastructure such as router, mobile station and base station. Each MN can move in the network anywhere and anytime. So the arrangement of the MNs in the network is changing continuously; therefore the topology is dynamic. The MNs have limited amount of energy, so it requires multi hop routing to send the packet to the destination. The size of the MANET is small due to the limited amount of energy. Moreover, it does not have any centralized node and is useful for the purpose where there is no infrastructure or infrastructure gets destroyed such as disaster prone areas or military fields [1].

Unidirectional or asymmetry links in MANET is caused due to energy depletion of the nodes with the time and hence the transmission range also decreases. As shown in Fig. 1, there is a bidirectional link between $S$ and $P$. Similarly, there is a bidirectional link between $\mathrm{Q}$ and $\mathrm{P}$. It means that $\mathrm{P}$ can send routing update message to $S$ and $Q$ and can also receive routing update message from $\mathrm{S}$ and $\mathrm{Q}$. Initially the energy of $\mathrm{P}$ is $\mathrm{w}$ watts and transmission range is $\mathrm{R}$ units of distance. As some time passes energy of $\mathrm{P}$ depletes to w' watts and also transmission range decreases to $\mathrm{R}$ ' units of distance. But the energy of $\mathrm{S}$ and $\mathrm{Q}$ is conserved .Now $\mathrm{P}$ can send the routing update message only to $\mathrm{Q}$ as $\mathrm{S}$ does not lie in the transmission range of $\mathrm{P}$. S and $\mathrm{Q}$ can still send the routing update message to $\mathrm{P}$. Therefore, the link between $\mathrm{P}$ and $\mathrm{S}$ becomes unidirectional i.e. $\mathrm{S} \rightarrow \mathrm{P}$ due to depletion of energy [2].

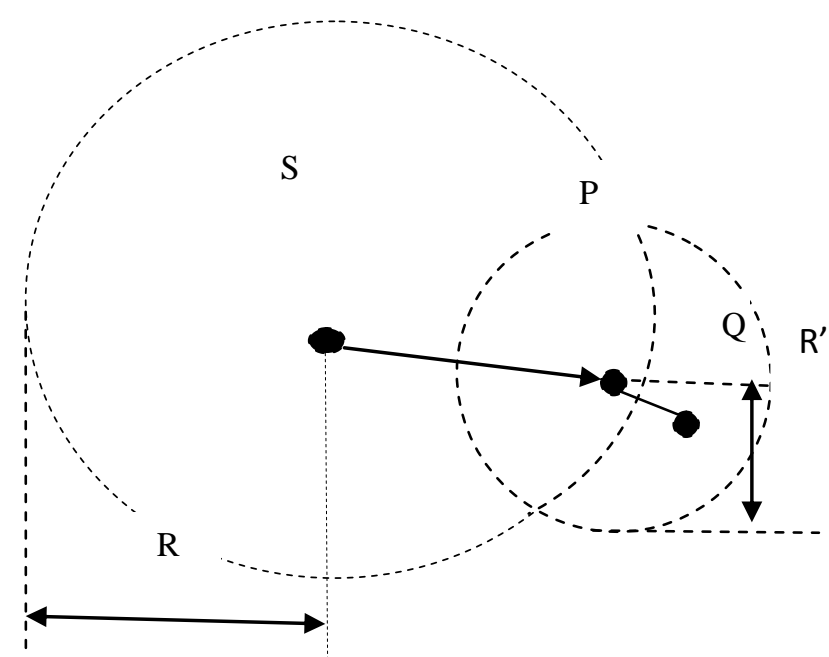

Figure 1. Presence of unidirectional links due to energy depletion

Protocols have been classified into table driven protocols and source-initiated on demand driven protocols. Table driven protocols maintain up to date routing information and send the routing update message after a fixed interval of time. Source initiated on demand protocols produces routes to the destination when demanded by the source. Destination Sequence Distance Vector (DSDV) is a table driven protocol which is adapted from a Bellman Ford algorithm. Each node in DSDV has routing table that contains sequence numbers ( $\mathrm{SN}$ ), destination's address, next hop and number of hops required to reach the final destination. The $\mathrm{SN}$ is used to distinguish the stale and broken routes from the new routes. SN helps to determine how old the route is. DSDV does not work for unidirectional links because the routing update messages are lost in asymmetric network [3].

In Fig. 2 the link between $\mathrm{P}$ and $\mathrm{Q}$ is unidirectional i.e. from $\mathrm{P} \rightarrow \mathrm{Q}$. $\mathrm{P}$ can send route updates to $\mathrm{Q}$ but $\mathrm{Q}$ cannot send the route update to $\mathrm{P}$. Therefore, $\mathrm{P}$ would never receive routing update messages from $\mathrm{Q}$ and will never come to know that there is a path to $\mathrm{R}$ via $\mathrm{Q}$. By finding reverse path, the problems with unidirectional links could be solved. 


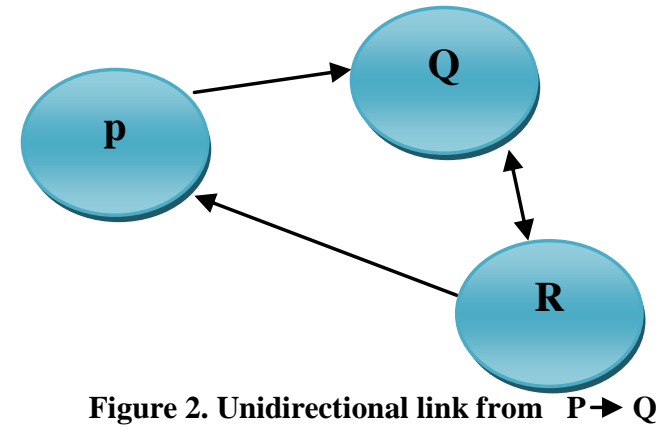

In this paper, the proposed model U-DSDV works efficiently in unidirectional links. It uses the concept of reverse distributed Bellman Ford algorithm for finding and maintaining the multihop reverse routes to each unidirectional link.

The remainder of this paper is organized as follows: Section II discusses the previous works and provides the motivation for a new model to overcome the issues related to the asymmetric networks. U-DSDV is described in detail in Section III. Finally section IV concludes the paper and present future work in section $\mathrm{V}$.

\section{RELATED WORK}

In [3], Gouyou et al described about DSDV based on distance vector protocol. Each node maintains a routing table which consists of destination's address, number of hops to reach the destination node, next hop to reach the destination and SN. The difference between the stale and the new routes is determined by the SN. The SN of the broken links is odd otherwise it is even. There are two ways of sending routing updates: full dump and incremental. In full dump, complete routing table is sent to the destination and in incremental only the updated entries are shared to the neighbours. DSDV protocols suffer from various problems in case of unidirectional links. The presence of sink node becomes difficult to determine as the sink node cannot broadcast its presence to other nodes in the network. Thus, each node needs to keep a record of sufficient information to know about unidirectional link [9], [10], [18], [22].

In [4], Hu Hefei and Yuan Dongming proposed A-DSDV routing protocol for asymmetric mesh network. It determines the reverse route for the unidirectional links in the mesh networks. Reverse route to the destination is found by sending Route Request message to the neighbours. Whenever a source node gets ROUTE-UPDATE it does not immediately update its routing table instead it floods ROUTE-REQUEST to its neighbour. Furthermore, immediate nodes or the destination node sends ROUTE-RESPONSE to source node to update the routing table of the source node. As a result, A-DSDV is able to detect reverse route in the asymmetric mesh network.

In [5], Ramasubramanian et al proposed sub routing laye (SRL).It is a framework for providing the reverse path for unidirectional links. Reverse Bellman Ford Algorithm is used to find the reverse path when unidirectional link is present. It works by broadcasting "hello messages" to the nodes and determines the status of the link. Reverse route information is maintained by the SRL layer in addition to the list of in-neighbour of all the nodes. SRL receives the route reply packet from the nodes and uses this information to send the route reply to the destination using the reverse route when it encounters unidirectional link. Moreover, acknowledgement can also be send by the reverse route mechanism [6], [7].

An advanced message scheme is discussed in [11] to minimize the loss that happens due to the link breakage. It uses the existing information to determine the alternative loop free route to the destination; hence minimizes the packet delivery loss. The main characteristics of the wireless network have been discussed in [8], [16]. It also summarizes the performance analysis of the packet data delivery under various conditions.

A tunnelling mechanism for the unidirectional links in the MANET is given in [17]. It also detailed about the scenarios that lead to the unidirectional links in the network such as hidden terminal and transmission power disparity. Subsequently, tunnelling approach is presented as a solution in order to overcome the issues of knowledge and routing asymmetry. The wide range of routing protocol and their comparative analysis is presented in [12], [13], [14], [15], [19], [21]. Moreover, a proactive routing protocol for the wireless networks is given in [20].

A new framework is proposed for the asymmetric links in the network [23]. It builds multi-hop reverse routes for the unidirectional links and provides better connectivity; therefore improving the performance. However, the use of DSDV in this framework results in periodic updates that are nevertheless to the changes in the topology. Consequently, to overcome the issues related to the existing methods of unidirectional links, section III discusses a new model to provide solution for the limited connectivity problem.

\section{U-DSDV PROPOSED MODEL}

The U-DSDV proposed model is designed to overcome the problem of limited connectivity due to the presence of unidirectional asymmetric links in the network. The proposed algorithm is the modified version of DSDV and also finds the reverse path. It uses the concept of bidirectional routing abstraction along with in-neighbour and out-neighbour is used.

Each node in the network sends a packet that helps to determine the in-neighbour of 1 hop count. Fig. 3 illustrates the packet format. Nodes that receive the packet inferred that the sender node is 1 hop in-neighbour of the receiver node.

\begin{tabular}{|l|l|}
\hline Source_node & Hop count(1) \\
\hline
\end{tabular}

Figure 3. Packet format

If there is a link from ' $A$ ' to ' $B$ ', then ' $A$ ' is the 1 hop inneighbour of ' $B$ '. In case of multi-hop path from ' $A$ ' to ' $B$ ' e.g.: $\mathrm{A}-\mathrm{C} \rightarrow \mathrm{B}$, then ' $\mathrm{A}$ ' is considered as 2 hops in-neighbour of ' $\mathrm{B}$ '. Fig. 4 demonstrate the format of the table. First three columns of the tables represent the list of out-neighbours. This also helps to find the path to the destination node in asymmetric unidirectional links. Next three columns represent the list of in-neighbours which are useful for determining the reverse path.

\section{Notations:}

Out: node that have path from the source node

In: node that have path to the source node

Ohop: hop-count of out-neighbour

Ihop : hop count of in-neighbour

Opre : immediate predecessor of destination for Out

Ipre: immediate predecessor of source for In

$\mathrm{N}$ : node in the network 


\begin{tabular}{|l|l|l|l|l|l|}
\hline Out & Ohop & Opre & In & Ihop & Ipre \\
\hline
\end{tabular}

Figure 4. Table parameters

The pseudo-code of the proposed algorithm is shown in Figure.5.

Proposed algorithm for asymmetric unidirectional links:

Each $\mathrm{N}$ broadcast the packet.(Fig.1)

if $\mathrm{N}$ receives the broadcasted packet

$\mathrm{N}$ is 1hop out-neighbour of the source $\mathrm{N}$

Source $\mathrm{N}$ is 1hop in-neighbour of $\mathrm{N}$

else

$\mathrm{N}$ is not 1hop out-neighbour of source $\mathrm{N}$.

Source $\mathrm{N}$ is not the 1 hop in-neighbour of $\mathrm{N}$.

Each $\mathrm{N}$ maintains a routing table. (Fig.4)

Each $\mathrm{N}$ share its table with the 1hop out-neighbours.

Each $\mathrm{N}$ that receives the table generates a modified table.

New out- neighbours of more than 1hop count

New in-neighbours of more than 1hop count.

Distributed Bellman-ford algorithm is used to select the shortest path from in-neighbours and to out- neighbours.

Steps [5-7] are repeated for all the nodes in the network.

Routing table of each $\mathrm{N}$ has the reachable path to outneighbour and the reverse path to itself.

\section{Figure 5. Proposed algorithm}

Following is the precise definition of U-DSDV through an example based on the steps presented in Fig.5.

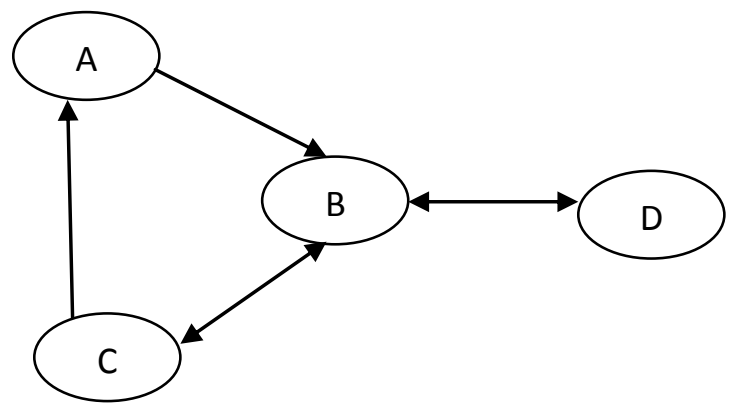

Figure 6. Example of unidirectional asymmetric link

Initial table for each node:

Table 1 Routing Table for $\mathrm{A}$

\begin{tabular}{|c|c|c|c|c|c|}
\hline Out & Ohop & Opre & In & Ihop & Ipre \\
\hline B & 1 & A & C & 1 & C \\
\hline & & & & & \\
\hline
\end{tabular}

Table 2 Routing Table for $B$

\begin{tabular}{|c|c|c|c|c|c|}
\hline Out & Ohop & Opre & In & Ihop & Ipre \\
\hline C & 1 & B & A & 1 & A \\
\hline D & 1 & B & C & 1 & C \\
\hline & & & D & 1 & D \\
\hline
\end{tabular}

Table 3 Routing Table for $\mathrm{C}$

\begin{tabular}{|c|c|c|c|c|c|}
\hline Out & Ohop & Opre & In & Ihop & Ipre \\
\hline A & 1 & C & B & 1 & B \\
\hline B & 1 & C & & & \\
\hline
\end{tabular}

Table 4 Routing Table for $D$

\begin{tabular}{|c|c|c|c|c|c|}
\hline Out & Ohop & Opre & In & Ihop & Ipre \\
\hline B & 1 & D & B & 1 & B \\
\hline & & & & & \\
\hline
\end{tabular}

2. Node ' $B$ ' can send its table to its 1 hop out-neighbours ' $C$ ' and ' $D$ '. Node ' $C$ ' came to know that it has 2 hops out-neighbour ' $D$ ' and node ' $\mathrm{D}$ ' came to know that it is reachable from ' $\mathrm{A}$ ' and ' $\mathrm{C}$ ' at 2 hops count through ' $\mathrm{B}$ '. Modified tables are as follows:

Table 5 C's modified table

\begin{tabular}{|c|c|c|c|c|c|}
\hline Out & Ohop & Opre & In & Ihop & Ipre \\
\hline A & 1 & C & A & 2 & B \\
\hline B & 1 & C & B & 1 & B \\
\hline D & 2 & B & D & 2 & B \\
\hline
\end{tabular}

Table 6 D's modified table

\begin{tabular}{|c|c|c|c|c|c|}
\hline Out & Ohop & Opre & In & Ihop & Ipre \\
\hline B & 1 & D & A & 2 & B \\
\hline C & 2 & B & B & 1 & B \\
\hline & & & C & 2 & B \\
\hline
\end{tabular}

When the C's modified table is shared with its 1hop outneighbours, node ' $A$ ' concludes that it is reachable from ' $B$ ' using 2 hop through ' $\mathrm{C}$ '. This is the reverse path from ' $\mathrm{B}$ ' to ' $\mathrm{A}$ ' through ' $C$ '. ' $A$ ' also came to know that it is reachable to ' $D$ ' through ' $B$ ' i.e. path from ' $A$ ' to ' $D$ ' is of 2 hop count through node ' $\mathrm{B}$ '. 
Table 7 A's modified table:

\begin{tabular}{|c|c|c|c|c|c|}
\hline Out & Ohop & Opre & In & Ihop & Ipre \\
\hline B & 1 & A & B & 2 & C \\
\hline C & 2 & B & C & 1 & C \\
\hline D & 2 & B & D & 3 & C \\
\hline
\end{tabular}

As presented in the above steps, it can be concluded that each node can determine the shortest path to the destination and the reverse path from the unidirectional out-neighbours to the node itself. Additionally, SN let each node to determine the new route over the stale route. Basically, it is by each node and incremented by two for each update. When a node receives an update with higher $\mathrm{SN}$, the old value of the route is replaced restricting the occurrence of routing loops in the network [3].

The metric value of infinity is assigned to the broken links in the network. Any route through this link is assigned an infinite metric and an incremented odd SN. The odd SN can be generated by any node except the destination node and has a greater value than the previous one. Subsequently, this update is propagated through the entire network and each node (except the destination) modifies its metric value to infinity after receiving the update for the destination node [3].

\section{CONCLUSION}

The performance of most of the routing protocols can degrade in the presence of the unidirectional links in the networks. Hence, the new U-DSDV is proposed that can work in unidirectional links. Each node maintains a routing table which contains the list of out-neighbours and in-neighbours. The stored information of the routing tables let each node to determine the shortest path to the destination and to find the reverse path from the unidirectional out-neighbours to the node itself.

\section{FUTURE WORK}

Unidirectional links can occur in any network due to the presence of noise sources and different transmission range of different nodes. Such links can limit the performance of most of the routing protocols which consider all the wireless links in the network as bidirectional. Since the proposed algorithm is flexible in nature; it can be used with the other routing protocols and can be analyzed further to present the relative performance results.

\section{REFERENCES}

[1] Jia Lu, Bin Zhang, Gang Han, JunHui Wang, WenHua Dou, "A New Improvement On DSDV",2011 IEEE

[2] Ravi Prakash, "A Routing Algorithm for Wireless Ad Hoc Networks with Unidirectional Links", Wireless Networks 7, 617-625, 2001

[3] Guoyou He, "Destination-Sequenced Distance Vector (DSDV) Protocol", Networking Laboratory Helsinki University of Technology

[4] Hu Hefei, Liu Yuan'an, Yuan Dongming, Tang Bihua, “An Efficient A-DSDV Routing Protocol For Asymmetric Mesh Networks ", 2009 IEEE

[5] Venugopalan Ramasubramanian, Ranveer Chandra, Daniel Moss'e, "Providing a Bidirectional Abstraction for Unidirectional Ad Hoc Networks", 2002

[6] Venugopalan Ramasubramanian and Daniel Mossé, "BRA:
A Bidirectional Routing Abstraction for Asymmetric Mobile Ad Hoc Networks", IEEE/ACM TRANSACTIONS ON NETWORKING, VOL. 16, NO. 1, FEBRUARY 2008

[7] Archana.M , Gopinathan.B, "Abstraction and Efficiency for MANET using Reverse Bellman Ford Algorithm",Vol2 Issue 2 February 2013

[8] Shilpa Shukla, Shelja Sharma: Study \& Analysis of DSDV, AODV \& DSR; International Journal of Advanced Research in Computer and Communication Engineering Vol. 2, Issue 5, May 2013

[9] Khaleel Ur Rahman Khan, A Venugopal Reddy Rafi U Zaman, K. Aditya Reddy T Sri Harsha, "An Efficient DSDV Routing Protocol for MANET and its usefulness for providing InternetAccess to Ad Hoc Hosts”,2008,IEEE

[10] Nayan Ranjan Paul, Laxminath Tripathy and Pradipta Kumar Mishra : Analysis and Improvement of DSDV Protocol; IJCSI International Journal of Computer Science Issues, Vol. 8, Issue 5, No 1, September 2011

[11] Liu, Ting, and Kai Liu. "Improvements on DSDV in mobile ad hoc networks." Wireless Communications, Networking and Mobile Computing, 2007. WiCom 2007. International Conference on. IEEE, 2007.

[12] Diamantopoulos, Fotis, and Anastasios A. Economides. "A performance study of DSDV-based CLUSTERPOW and DSDV routing algorithms for sensor network applications." Wireless Pervasive Computing, 2006 1st International Symposium on. IEEE, 2006.

[13] Mutalik, Pramod, et al. "A comparative study on AODV, DSR and DSDV routing protocols for Intelligent Transportation System (ITS) in metro cities for road traffic safety using VANET route traffic analysis (VRTA)." Advances in Electronics, Communication and Computer Technology (ICAECCT), 2016 IEEE International Conference on. IEEE, 2016.

[14] Aggarwal, Neha, et al. "Relative Analysis of AODV \& DSDV Routing Protocols for MANET based on NS2." Electrical, Electronics, and Optimization Techniques (ICEEOT), International Conference on. IEEE, 2016.

[15] Kumari, Neelu, et al. "New performance analysis of AODV, DSDV and OLSR routing protocol for MANET." Computing for Sustainable Global Development (INDIACom), 2016 3rd International Conference on. IEEE, 2016.

[16] Zhao, Jerry, and Ramesh Govindan. "Understanding packet delivery performance in dense wireless sensor networks." Proceedings of the 1st international conference on Embedded networked sensor systems. ACM, 2003.

[17] Nesargi, Sanket, and Ravi Prakash. "A tunneling approach to routing with unidirectional links in mobile ad-hoc networks." Computer Communications and Networks, 2000. Proceedings. Ninth International Conference on. IEEE, 2000.

[18] De Couto, Douglas SJ, et al. "Performance of multihop wireless networks: Shortest path is not enough." ACM SIGCOMM Computer Communication Review 33.1 (2003): 83-88.

[19] Abolhasan, Mehran, Tadeusz Wysocki, and Eryk Dutkiewicz. "A review of routing protocols for mobile ad hoc networks." Ad hoc networks 2.1 (2004): 1-22. 
[20] Lee, Unghee, Scott F. Midkiff, and Jahng S. Park. "A proactive routing protocol for multi-channel wireless ad-hoc networks (DSDV-MC)." Information Technology: Coding and Computing, 2005. ITCC 2005. International Conference on. Vol. 2. IEEE, 2005.

[21] Yang, Xin, et al. "Performance Optimisation for DSDV in VANETs." Modelling and Simulation (UKSim), 2015 17th UKSim-AMSS International Conference on. IEEE, 2015.

[22] Thamizhmaran, K., Akshaya Devi Arivazhagan, and M.
Anitha. "Co-operative analysis of proactive and reactive protocols using Dijkstra's Algorithm." Intelligent Systems and Control (ISCO), 2015 IEEE 9th International Conference on. IEEE, 2015.

[23] Bali, Vikram, et al. "A Framework to Provide a Bidirectional Abstraction of the Asymmetric Network to Routing Protocols." Emerging Trends in Engineering and Technology (ICETET), 2009 2nd International Conference on. IEEE, 2009. 